\title{
Superfici di interesse culturale geo-paleontologico con orme di dinosauro del Cretaceo (Albiano superiore): l'esempio di Lama Balice nella Città Metropolitana di Bari
}

\author{
Marco Petruzzelli (1), Stefano Cardia (1), Antonietta Cilumbriello (2), Ruggero Francescangeli (1), \\ Rafael La Perna (1), Maria Marino (1), Antonella Marsico (1), Fabio Massimo Petti (1, 3), Luisa Sabato (1, 4), \\ Luigi Spalluto (1), Eliana Stigliano (1) \& Marcello Tropeano (1)
}

\footnotetext{
(1) Dipartimento di Scienze della Terra e GeoAmbientali, Università degli Studi di Bari Aldo Moro, via Orabona 4, 70125 Bari (Italy).

(2) Genzano di Lucania (PZ) (Italy).

(3) MUSE - Museo delle Scienze, Corso del Lavoro e della Scienza 3, 38122 Trento (Italy).

(4) Dipartimento di Biologia, Università degli Studi di Bari Aldo Moro, via Orabona 4, 70125 Bari (Italy).

Corresponding author e-mail: luisa.sabato@uniba.it
}

Document type: Article.

Manuscript received 07 September 2019; accepted 11 November 2019; editorial responsibility and handling by F. Cifelli.

\section{ABSTRACT}

Along the eastern flank of the Murge, a karst plateu located in Apulia (southern Italy), the "Parco Naturale Regionale Lama Balice" ("Lama Balice" Regional Natural Park), which falls into the Metropolitan City of Bari, was established; in the Park, on a bottom surface of an abandoned quarry, several dinosaur footprints were found. The quarry develops in the Cretaceous limestones of the Calcare di Bari Fm, and shows an about $20 \mathrm{~m}$ thick upper Albian succession made up of carbonates and dolomites. A Research Convention stipulated between the Park authority, the University of Bari and the "Soprintendenza Archeologia, Belle Arti e Paesaggio per la Città Metropolitana di Bari", allowed the access to the quarry and the study of both the stratigraphic succession and the trumpled surface.

In order to investigate the outcropping succession, a stratigraphic log was realised, and 54 samples were collected. Thin sections were produced and a facies analysis was carried out by means of macroscopic feature observations and microscopic ones. Several facies were recognised allowing to attribute the studied succession to a tida flat frequently subject to prolonged subaerial exposure. The study of the surface consisted both in an ichnological analysis of individual footprints and in the identification of potential trackways. In order to highlight the main morphological features of footprints, an ichnological survey was realised by means of interpretative drawings carried out directly in situ. Then, data were processed digitally through photos and photomosaics. The best-preserved footprints allowed us to recognize the presence of theropods, ankylosaurs, and probably sauropods. All the recognized taxa belong to small- to medium-sized dinosaurs.

The presence of dinosaur footprints represents an important contribute to the knowledge of Cretaceous paleoenvironments of this sector of the Apulia Carbonate Platform corresponding to the Murge region.

Interestingly, the occurrence of dinosaur footprints within a park area, located close to a large city and an airport, represents a powerful educational and cultural tool.

KEYWORDS: Apulia, Cretaceous, dinosaurs, carbonate platform, tidal flat.

\section{INTRODUZIONE}

Le segnalazioni di superfici ad orme di dinosauro, riconosciute nella successione di piattaforma carbonatica cretacea delle Murge, sono ormai numerose e significative
(Nicosia et al., 2000a; 2000b; Iannone, 2003; Petruzzelli, 2008; 2017; Sacchi et al., 2009; Petti et al., 2010; 2018; Petruzzelli et al., 2012; 2019). Questa regione corrisponde al noto altopiano carsico pugliese contraddistinto da piccole incisioni fluviali in roccia, localmente chiamate "lame". Le lame, che in alcuni tratti presentano pareti subverticali e fondo piatto, a luoghi ricoperto da un materasso detritico poco spesso, sono generalmente secche, con un'attività effimera indotta esclusivamente da eventi di piovosità estrema. Queste caratteristiche hanno reso le pareti delle lame favorevoli all'apertura di cave ad anfiteatro, alcune delle quali con superfici di lavoro prossime al livello di base dei corsi d'acqua e frequentemente alluvionate durante eventi di piena (Moretti, 2005; Mossa, 2007; Andriani \& Walsh, 2009; Gioia et al., 2011).

Uno di questi corsi d'acqua in roccia, caratterizzato dalla presenza di numerose cave lungo il suo alveo, è rappresentato dalla Lama Balice. Il settore della lama compreso fra l'abitato di Bitonto e la foce, a nord della città di Bari, è stato riconosciuto come area di interesse storico e naturalistico dalla Regione Puglia attraverso la L.R. n.15 del 2007, che ha istituito il "Parco Naturale Regionale Lama Balice". Il Parco si estende per circa 500 ettari e ricade completamente nell'area della Città Metropolitana di Bari (Fig. 1). La presenza di orme di dinosauro è stata segnalata più volte su superfici di lavoro di cave di prestito, ormai dismesse, aperte nella successione cretacea e ricadenti nell'area del Parco.

Al fine di dare corpo e peso scientifico a tali segnalazioni è stata firmata e portata a termine una convenzione per lo studio e la conservazione di una di tali superfici, ritenuta di particolare interesse culturale geo-paleontologico. I soggetti partecipanti alla convenzione sono stati: il Parco Naturale Regionale Lama Balice, l'Università degli Studi di Bari (Dipartimento di Scienze della Terra e Geoambientali) e la Soprintendenza Archeologia, Belle Arti e Paesaggio per la Città Metropolitana di Bari. Tale convenzione ha consentito il riconoscimento e lo studio 


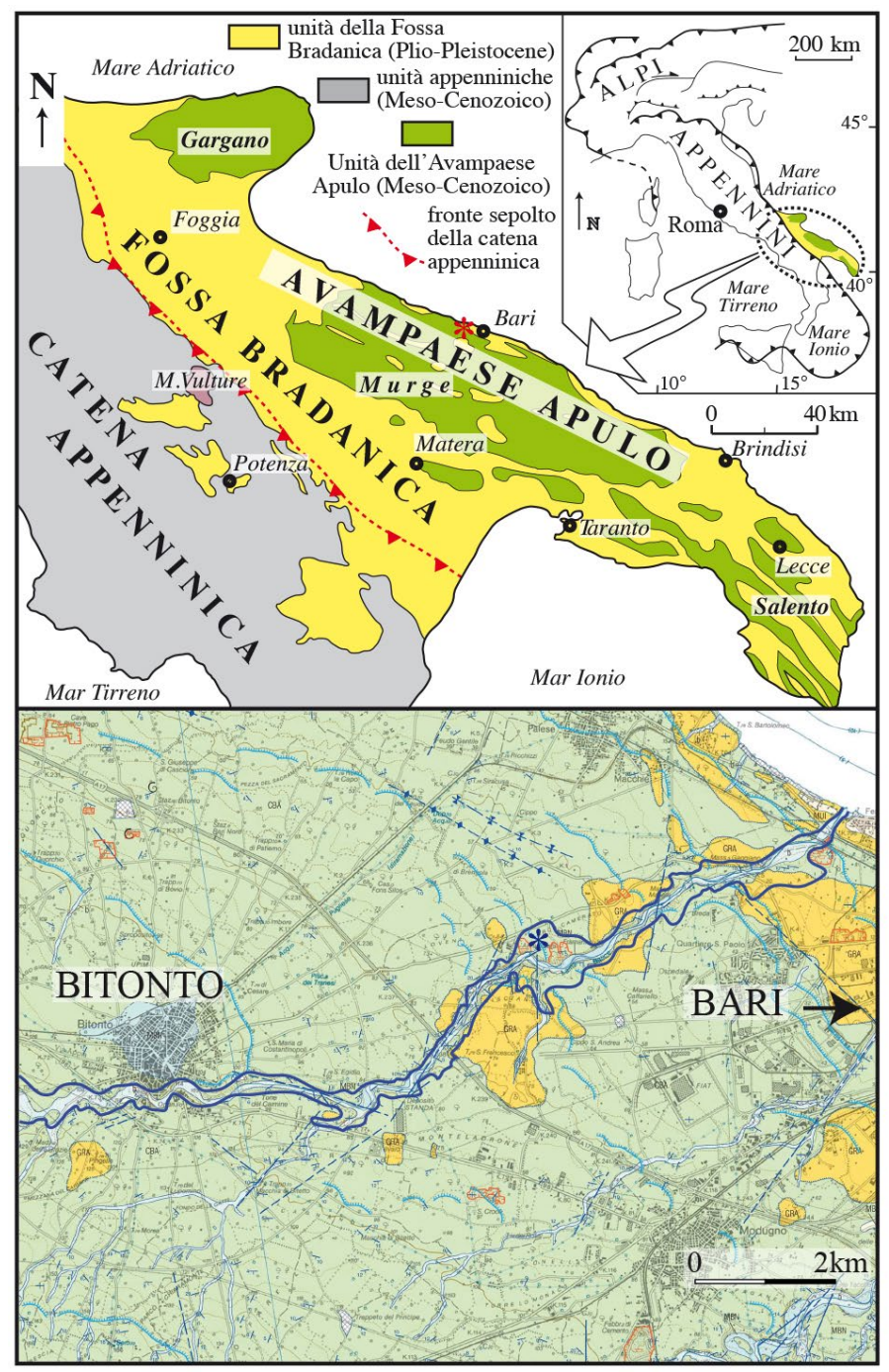

Fig. 1 - In alto: carta geologica schematica dell'Italia meridionale (Pieri et al., 1997, mod.); l'asterisco rosso indica l'ubicazione del Parco Naturale Regionale Lama Balice. In basso: stralcio del $F^{\circ} 438$ BARI in scala 1:50.000 (SERVIZIO GEOLOGICO D'ITALIA, 2011); l'area in blu indica l'ubicazione di circa il 90\% dell'area parco, l'asterisco blu indica l'ubicazione della ex-Cava SELP; in verde il Calcare di Bari (Cretaceo), in giallo la Calcarenite di Gravina (Pleistocene inferiore), in celeste chiaro i depositi subattuali delle lame.

di orme di dinosauro sulla superficie di fondo della "exCava SELP” (Francescangeli et al., 2019), già segnalata con codice CGP0254 nel volume "Geositi della Puglia" (Mastronuzzi et al., 2015) e argomento di due tesi di laurea inedite (Cardia, 2019; Stigliano, 2019). Oltre che della convenzione già richiamata, lo studio ha beneficiato anche di un finanziamento concesso dalla Fondazione Puglia al Dipartimento di Scienze della Terra e Geoambientali dell'Università di Bari per l'acquisto di uno strumento necessario alla realizzazione di sezioni sottili di roccia.

\section{INQUADRAMENTO GEOLOGICO}

Dal punto di vista geologico, l'area in cui ricade il Parco Naturale Regionale Lama Balice si colloca sul fianco orientale delle Murge. Queste, così come gran parte dell'attuale territorio pugliese, ricadono nel dominio geologico-strutturale dell'Avampaese Apulo (D'Argenio et al., 1973; Ricchetti et al., 1988) (Fig. 1), caratterizzato da un'imponente successione carbonatica mesozoica attribuita alla Piattaforma Carbonatica Apula (D'Argenio, 1974; Ricchetti, 1975; Pieri, 1980). Le vestigia di questa piattaforma corrispondono ai grandi ammassi rocciosi calcarei del Gargano, delle Murge e del Salento (Ricchetti et al., 1988). Nel suo complesso, la successione sedimentaria affiorante riferita alla Piattaforma Carbonatica Apula ha un'età che si estende dal Giurassico superiore a tutto il Cretaceo, interrotta da un ampio hiatus evidenziato da una discontinuità stratigrafica con bauxiti (Mongelli et al., 2015), compreso tra il Cenomaniano superiore ed il Turoniano. La successione affiorante nel territorio delle Murge, riferita esclusivamente al Cretaceo, è stata suddivisa in base alla presenza di questa discontinuità in due formazioni: il Calcare di Bari (Valanginiano-?Turoniano inferiore) e il Calcare di Altamura (Turoniano superioreMaastrichtiano) (Valduga, 1965; Azzaroli \& Valduga, 1967; Ciaranfi et al., 1988; Spalluto et al., 2005).

La ex-Cava SELP, compresa nel perimetro del Parco Lama Balice e che presenta una superficie con orme di dinosauro, si apre nei depositi cretacei del Calcare di Bari (Valduga, 1965; Azzaroli \& Valduga, 1967; Ricchetti, 1975). L'area su cui insiste il Parco ricade nel Foglio Geologico 438 "Bari" in scala 1:50.000 (Servizio Geologico d'Italia, 2011) (Fig. 1), e la porzione del Calcare di Bari affiorante nell'ambito del Foglio 438 è stata riferita all'Albiano inferiore p.p.-Cenomaniano superiore (Spalluto \& Caffau, 2010; Servizio Geologico d'Italia, 2011; Pieri et al., 2011a; 2011b; Spalluto, 2012). In particolare, in base alla sua posizione stratigrafica, la successione affiorante nella ex-Cava SELP può essere riferita alla parte terminale dell'Albiano superiore (Francescangeli et al., 2019).

Il primo ritrovamento di orme di dinosauro nella successione carbonatica cretacea delle Murge fu effettuato su una superficie di cava esposta nei pressi di Altamura (Nicosia et al., 2000b). Successivamente, altri livelli con orme di dinosauro sono stati scoperti in depositi mesozoici affioranti in diversi altri siti pugliesi. Tali evidenze hanno suggerito che la Piattaforma Carbonatica Apula, seppur fiancheggiata da bacini marini profondi, potesse conservare un collegamento con le principali masse continentali (Laurasia e Gondwana) (Bosellini, 2002; Conti et al., 2005; Petti, 2006; Nicosia et al., 2007; Petti et al., 2008; Zarcone et al., 2010).

\section{ANALISI DI FACIES}

L'analisi di facies è stata condotta lungo le pareti dell'ex-Cava SELP, che si apre parzialmente ad anfiteatro partendo dalle pareti di Lama Balice e si approfondisce a pozzo sul fianco sinistro della lama stessa. La cava è ubicata in prossimità della strada SP 156 "BitontoAeroporto di Bari-Palese", alle spalle della Chiesa rurale dell'Annunziata.

La successione affiora lungo le pareti della cava per uno spessore complessivo di circa $20 \mathrm{~m}$ ed è formata da calcari in strati pressoché orizzontali, a luoghi con superfici blandamente ondulate (Fig. 2). Poiché la parte alta della successione è costituita da dolomie, lo studio si è concentrato nei primi $8,5 \mathrm{~m}$ circa (comprensivi di 


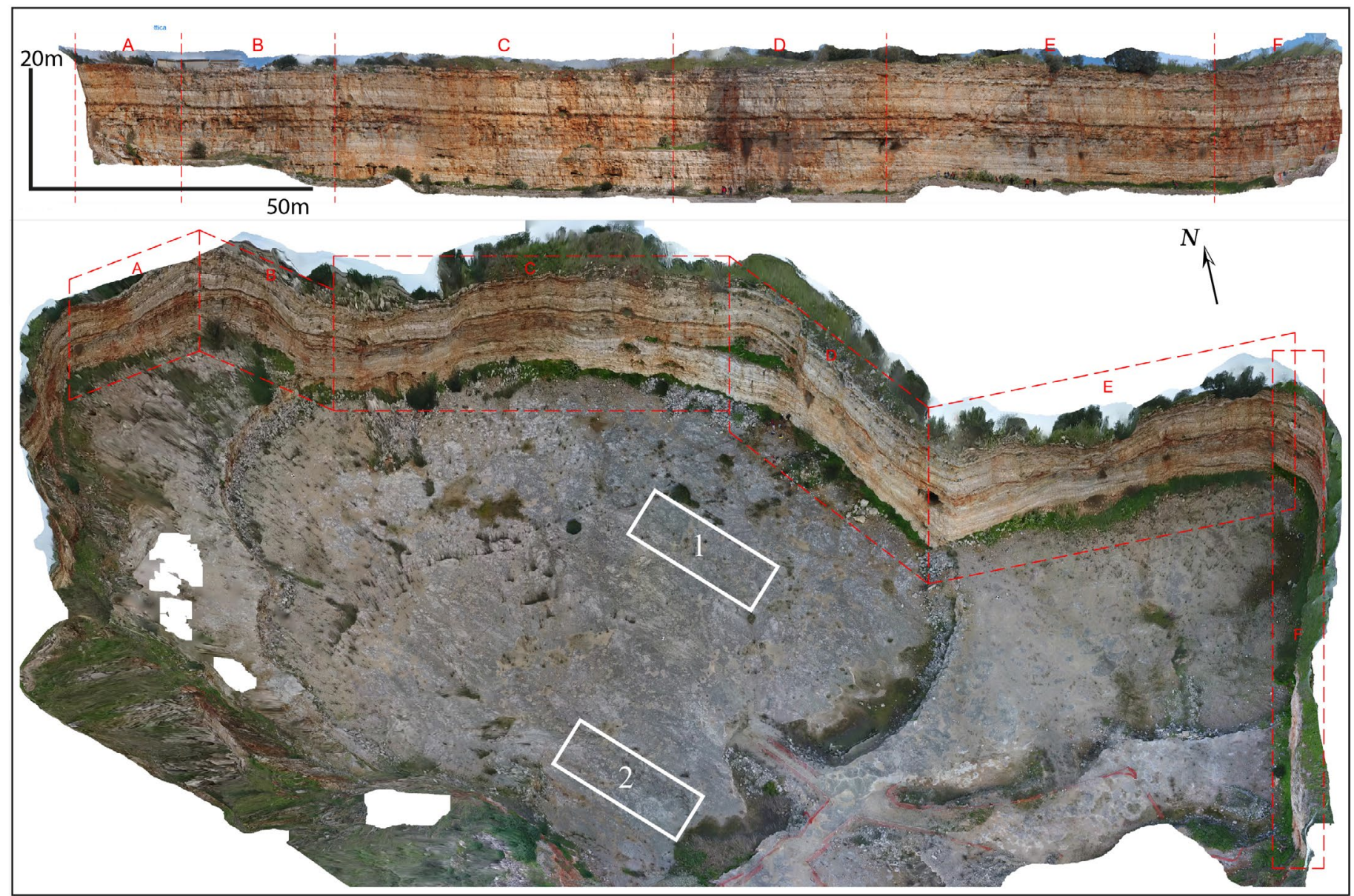

Fig. 2 - Rappresentazione 3D della ex-Cava SELP con fotomosaico delle pareti utilizzato per misure litostratigrafiche. Si noti l'andamento suborizzontale degli strati e la loro continuità laterale. Sul piano di cava, i rettangoli in bianco indicano le Aree 1 e 2 sulle quali è stata eseguita la pulizia manuale e lo studio icnologico di dettaglio.

0,50 m sottostanti la superficie di fondo cava con orme di dinosauro) con la realizzazione di un log stratigrafico (Fig. 3). Allo scopo di definire i caratteri delle microfacies, sono stati prelevati 54 campioni di roccia, dai quali sono state poi realizzate altrettante sezioni sottili, analizzate al microscopio ottico e descritte nel dettaglio, seguendo la classificazione di Dunham (1962).

La successione analizzata (Fig. 3) è rappresentata da mudstone in strati spessi alcune decine di centimetri, spesso bioturbati o fittamente laminati, a cui si intercalano strati da centimetrici a decimetrici di bindstone microbialitici. La successione è inoltre caratterizzata dalla presenza di livelli anche metrici di wackestone brecciati; localmente, a varie altezze, si osservano strati di alcune decine di centimentri di grainstone, wackestone e packstone intrabiopeloidali.

Di seguito si riportano sinteticamente le principali tessiture, riconosciute sia sulla base delle osservazioni macroscopiche sul terreno che dall'osservazione delle microfacies, e le relative interpretazioni paleoambientali.

Grainstone intrabiopeloidale laminato: questa microfacies è costituita da granuli sub-arrotondati rappresentati da foraminiferi bentonici, frammenti di gusci di bivalvi, alghe calcaree, intraclasti e peloidi distribuiti su lamine. I peloidi derivano dalla completa micritizzazione dei bioclasti (Tav. 1A, B). L'ambiente di riferimento è quello subtidale corrispondente ad una laguna a circolazione aperta soggetta all'azione di onde e correnti.
- Packstone intrabiopeloidale: questa microfacies è costituta da abbondanti peloidi, intraclasti micritici, alghe calcaree, piccoli foraminiferi bentonici e frammenti di gusci di bivalvi con un sottile cortice micritico. Così come per la precedente microfacies, i peloidi derivano dalla micritizzazione dei bioclasti (Tav. 1C, D). L'ambiente di sedimentazione è quello subtidale aperto, anche se la presenza di matrice indica condizioni di basso idrodinamismo.

- Wackestone biopeloidale: questa microfacies a tessitura fango-sostenuta è costituita da foraminiferi bentonici, alghe calcaree e peloidi (Tav. 1E, F). L'ambiente di sedimentazione è quello subtidale corrispondente a una laguna di bassa energia.

- Mudstone/wackestone biopeloidale bioturbato: questa microfacies è costituita da ostracodi, piccoli foraminiferi bentonici (miliolidi) e peloidi. In particolare, i peloidi sono concentrati all'interno delle tane degli organismi limivori e sono di probabile origine fecale (fecal pellets) (Tav. 1G, $\mathrm{H})$. L'ambiente di sedimentazione è relativo a condizioni subtidali, corrispondenti a una laguna con circolazione ristretta delle acque.

- Mudstone fittamente laminato con piccole cavità del tipo birdseye: questa microfacies è costituita da calcari micritici fittamente laminati, di probabile origine microbialitica, con piccole cavità millimetriche circolari, ovali o irregolari riempite da calcite spatica (Tav. 2A, B). 


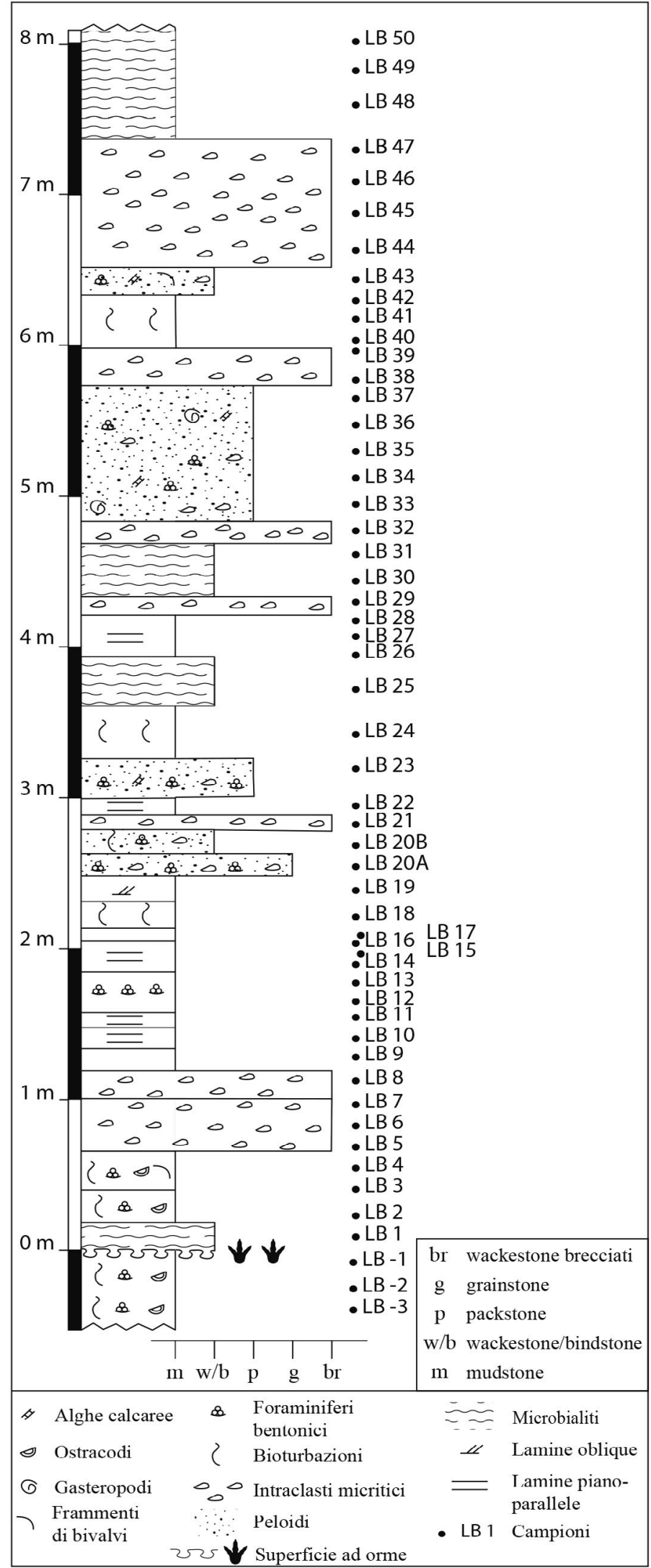

Fig. 3 - Log stratigrafico di sintesi della parte bassa della successione affiorante nella ex-Cava SELP. Il livello a $0 \mathrm{~m}$ rappresenta la superficie di fondo cava, sulla quale sono impresse le orme di dinosauro. Al fine di caratterizzare le facies immediatamente al di sotto della superficie ad orme, in una piccola cavità naturale presente sul fondo della cava sono stati prelevati 3 campioni dai sottostanti $55 \mathrm{~cm}$.
L'ambiente di sedimentazione è riferibile ad una piana intertidale/supratidale.

- Bindstone stromatolitico con numerose cavità del tipo birdseye: questa litofacies è costituita da un'alternanza di lamine sparitiche ricche in peloidi e bioclasti alternate a lamine micritiche scure. La presenza dei birdseye è più abbondante rispetto alla precedente litofacies (Tav. 2C, D). I caratteri di facies sono riferibili ad un ambiente di sedimentazione intertidale/supratidale.

- Mudstone con cavità di tipo stromatactis: si tratta di calcari micritici costituiti da numerose cavità ampie da alcuni millimetri a pochi centimetri del tipo stromatactis con riempimenti geopetali. In particolare, la parte inferiore delle cavità è costituita da silt di origine vadosa, mentre la parte superiore è riempita da calcite spatica (Tav. 2E, F). La sedimentazione di questa facies è avvenuta in un ambiente subtidale ristretto, mentre le cavità con i riempimenti geopetali si sono formate in seguito ad esposizione in ambiente subaereo.

- Wackestone brecciati: questa litofacies è costituita da intraclasti micritici di dimensioni variabili, da alcuni millimetri ad alcuni centimetri, delimitati da numerose fratture da disseccamento riempite da calcite spatica e, nella parte superiore della successione studiata, da dolomite (Tav. 2G, H). L'ambiente di sedimentazione di questa litofacies è attribuibile ad una piana supratidale soggetta a un prolungato disseccamento.

Queste facies si ripetono lungo la successione analizzata, suggerendo variazioni nei paleoambienti da subtidali, prima a circolazione aperta e poi ristretta, fino ad intertidali passanti a supratidali, frequentemente soggetti a una prolungata esposizione subaerea.

\section{LA SUPERFICIE AD ORME DI DINOSAURO}

La superficie di fondo dell'ex-Cava SELP è stata analizzata con dettaglio, dopo aver effettuato un'accurata pulizia manuale di alcune zone, inizialmente coperte da detrito e da vegetazione. Tale operazione ha consentito di individuare due aree, ognuna ampia circa $300 \mathrm{mq}$, disposte una a nord (Area 1) e una a sud (Area 2) della superficie (Fig. 2), che mostravano maggiore presenza di orme, sia isolate che organizzate in piste.

Lo studio delle orme osservate in tali aree si è sviluppato in due fasi. La prima fase è consistita nella realizzazione di disegni interpretativi di ogni singola orma, tramite l'utilizzo di gessi da lavagna, allo scopo di evidenziare in via preliminare la forma e le caratteristiche morfologiche più salienti. Nella seconda fase sono stati raccolti tutti i parametri morfometrici, quando misurabili, come ad esempio larghezza e lunghezza dell'orma, numero delle dita, angolo tra le dita, impressione dei cuscinetti falangeali ed eventuali tracce di artigli e/o zoccoli. Si è cercato poi di riconoscere l'eventuale presenza di piste e, quando individuate, sono state effettuate le misurazioni dei caratteri principali, quali passo, doppio passo, angolo del passo, lunghezza, direzione e larghezza della pista, rapporti dimensionali tra orme della mano e del piede (Indice di eteropodia = Area impressa dalla mano/Area impressa dal piede; Lockley et al., 1994). La seconda fase è consistita nel ricalco delle stesse orme su teli di plastica trasparente, con particolare attenzione a quelle 

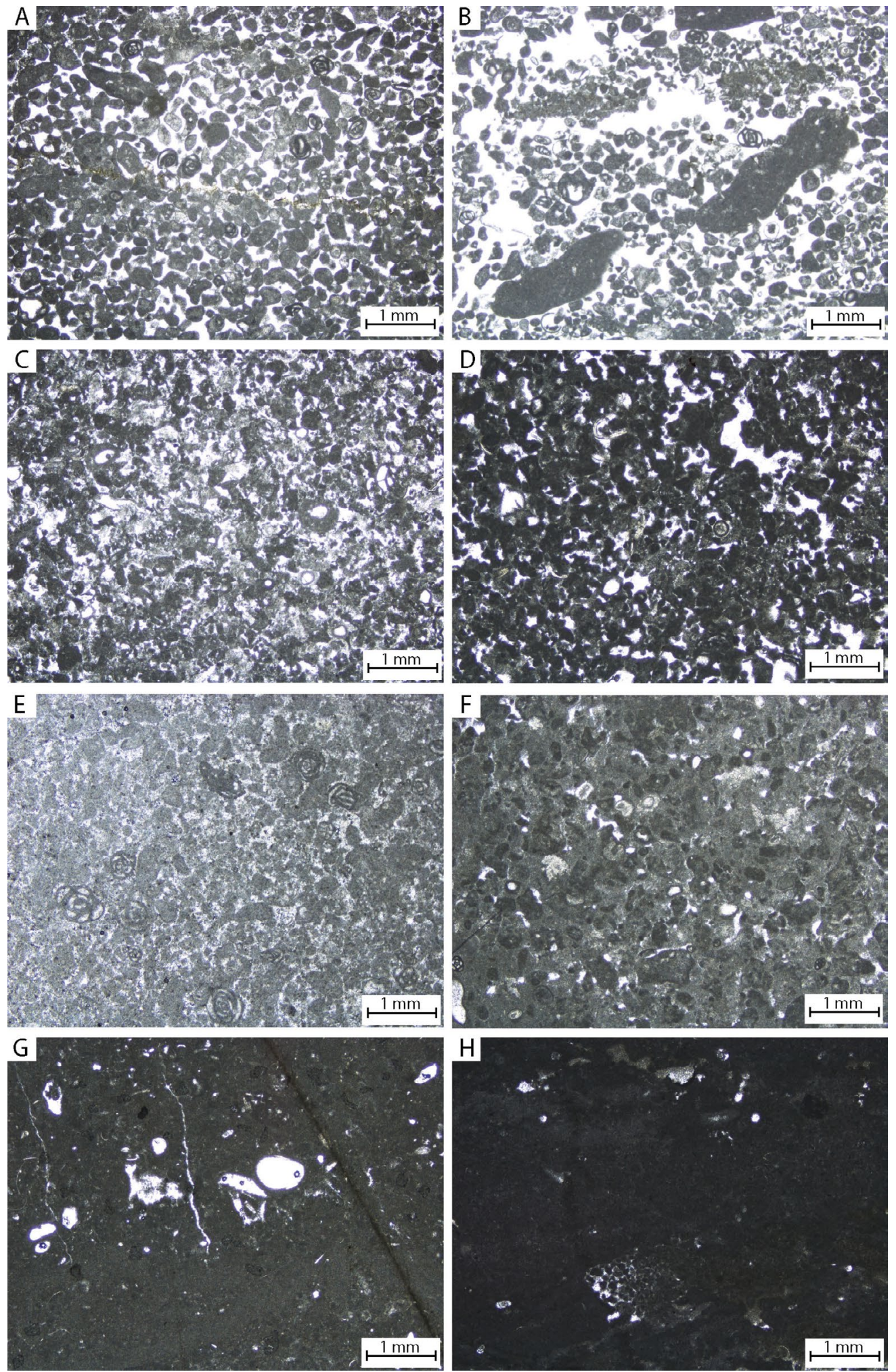

Tavola 1 - A) Grainstone intrabiopeloidale laminato. In sezione sottile si distinguono numerosi foraminiferi bentonici, intraclasti micritici e peloidi. I peloidi sono il prodotto della completa micritizzazione dei foraminiferi. B) Grainstone intrabiopeloidale laminato. Si distinguono intraclasti micritici sub-arrotondati, foraminiferi bentonici e peloidi. Gli spazi intergranulari sono stati completamente riempiti da calcite spatica. C) Packstone biopeloidale. Si distinguono numerose alghe calcaree, foraminiferi bentonici e peloidi. D) Packstone intrabiopeloidale. Si distinguono intraclasti micritici, peloidi, foraminiferi bentonici e alghe calcaree. E) Wackestone biopeloidale. Si distinguono abbondanti foraminiferi bentonici, alghe calcaree e peloidi, questi ultimi derivanti dalla completa micritizzazione dei bioclasti. F) Wackestone biopeloidale. Si distinguono alghe calcaree e peloidi di probabile origine fecale. G) Mudstone con gusci di ostracodi. H) Mudstone bioturbato. All'interno del fango carbonatico si distinguono tane di organismi infaunali riempite da peloidi. 

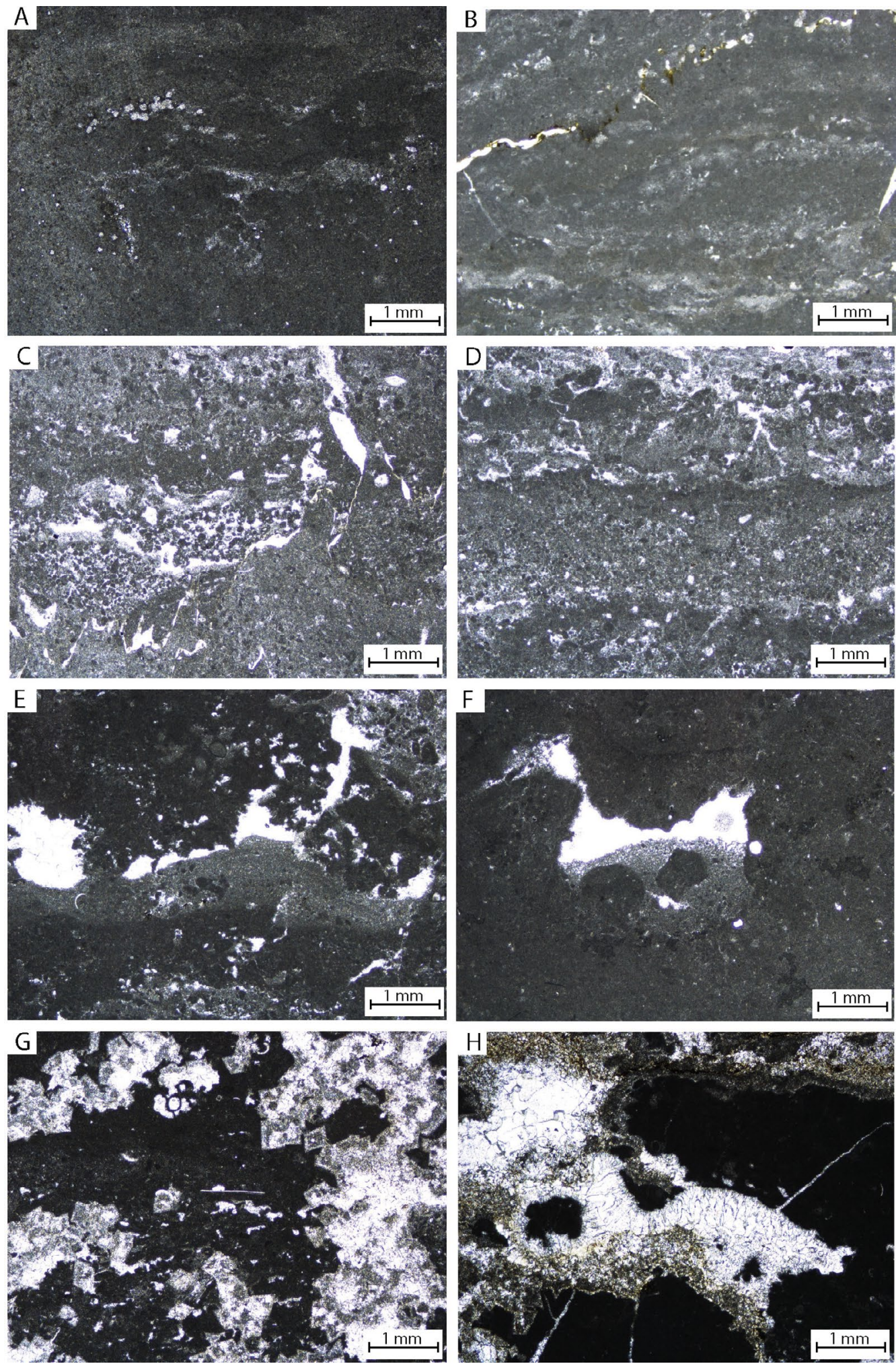

Tavola 2 - A) Mudstone laminato. B) Mudstone laminato. Si distinguono numerose lamine oblique irregolari scure di probabile origine microbialitica. C) Bindstone stromatolitico con stiloliti. Si distinguono lamine sparitiche ricche in peloidi e bioclasti con abbondanti cavità da disseccamento (birdseyes). D) Bindstone stromatolitico. Si distingue un'alternanza di lamine scure a granulometria fine e lamine peloidali più grossolane. Nella parte sommitale della sezione sottile si distinguono intraclasti e cavità prodotte dal disseccamento del fango carbonatico. E) Mudstone con cavità del tipo stromatactis Si distingue una cavità del tipo stromatactis ampia 5-6 mm con riempimento geopetale. In particolare, la parte inferiore delle cavità è costituita da silt vadoso mentre la parte superiore è riempita da calcite spatica. F) Mudstone con cavità del tipo stromatactis. G) Wackestone brecciato. Si distinguono intraclasti di dimensioni centimetriche. Negli spazi intergranulari è presente dolomite. H) Wackestone brecciato. Si distinguono intraclasti micritici irregolari di dimensioni centimetriche. Negli spazi intergranulari sono presenti riempimenti geopetali analoghi a quelli descritti per le cavità del tipo stromatactis. 
potenzialmente disposte in piste. I vari teli sono stati poi trasformati in formato digitale, tramite foto e creazione di un fotomosaico, realizzando un'immagine unica per ognuna delle due aree individuate (Fig. 4).

L'Area 1 (Fig. 2) è caratterizzata da numerose depressioni relativamente grandi, che sembrano a volte accoppiate in set mano-piede (Tav. 3p-r, Fig. 5).

Le misure rilevate per la coppia mano-piede meglio preservata indicano una lunghezza di $25 \mathrm{~cm}$ e una larghezza di $28 \mathrm{~cm}$ per il piede, e una lunghezza di $6 \mathrm{~cm}$ e una larghezza di $10 \mathrm{~cm}$ per la mano, evidenziando un forte indice di eteropodia. Si osservano inoltre 4 impressioni di dita nell'orma del piede, mentre non è possibile risalire al numero di quelle presenti nella mano (Fig. 5). L'altezza all'anca e la lunghezza totale, stimate seguendo la formula di Alexander (1976), sono di circa $1 \mathrm{~m}(\mathrm{~h}=4 * \mathrm{FL})$ e 4,5 m $(\mathrm{L} \approx 4,5 * \mathrm{~h})$, rispettivamente $(\mathrm{FL}=$ lunghezza del piede). Le caratteristiche osservate consentono di attribuire le orme sopra descritte a dinosauri quadrupedi del clade Ornithischia, probabilmente appartenenti al gruppo dei Thyreophora (?Anchylosauria).

Nonostante l'intenso grado di calpestio (degree of trampling o dinoturbation sensu Lockley \& Conrad, 1989), la cui densità è di circa il $40 \%$, e la frequente sovrapposizione dell'orma del piede su quella della mano, nell'Area 1 si può ipotizzare la presenza di tre possibili piste.

Altri tipi di orme rilevate nell'Area 1 sono invece dubitativamente attribuibili a dinosauri di più grandi dimensioni, possibilmente appartenenti al gruppo dei Sauropoda. Si tratta di coppie di orme profonde, rappresentate da un'orma particolarmente grande di forma ellissoidale (zampa posteriore) preceduta da un'orma più piccola a forma di mezzaluna (impressione della zampa anteriore) (Tav. 3m-o). Le misure effettuate su un'orma ben conservata della zampa posteriore sono di $45 \mathrm{~cm}$ di lunghezza e di $36 \mathrm{~cm}$ di larghezza. L'orma della zampa anteriore, mal preservata, risulta avere una dimensione dimezzata rispetto a quella posteriore. L'altezza all'anca stimata dell'animale che ha impresso quest'orma è circa $1,8 \mathrm{~m}(\mathrm{~h}=4 * \mathrm{FL})$, mentre la lunghezza è di circa $8 \mathrm{~m}$, misure stimate seguendo la formula di Alexander (1976).

L'Area 2 (Fig. 2), su cui è stato eseguito lo stesso tipo di studio dell'Area 1, mostra un più intenso grado di calpestio, con una densità di circa il 70\%, ma presenta una fortissima alterazione superficiale. In questa seconda area campione, la misura delle orme e l'osservazione dei loro dettagli sono risultate difficili, ma il loro range dimensionale conferma la presenza di due gruppi di dinosauri quadrupedi, compatibili con quelli indicati per la prima area. In particolare, considerate le grandi dimensioni di alcune orme, l'attribuzione a dinosauri sauropodi è ipotizzabile (Fig. 6). Il limitato areale e la cattiva qualità delle orme, che non preservano caratteristiche morfologiche diagnostiche sufficientemente affidabili, non hanno permesso l'individuazione certa di piste.

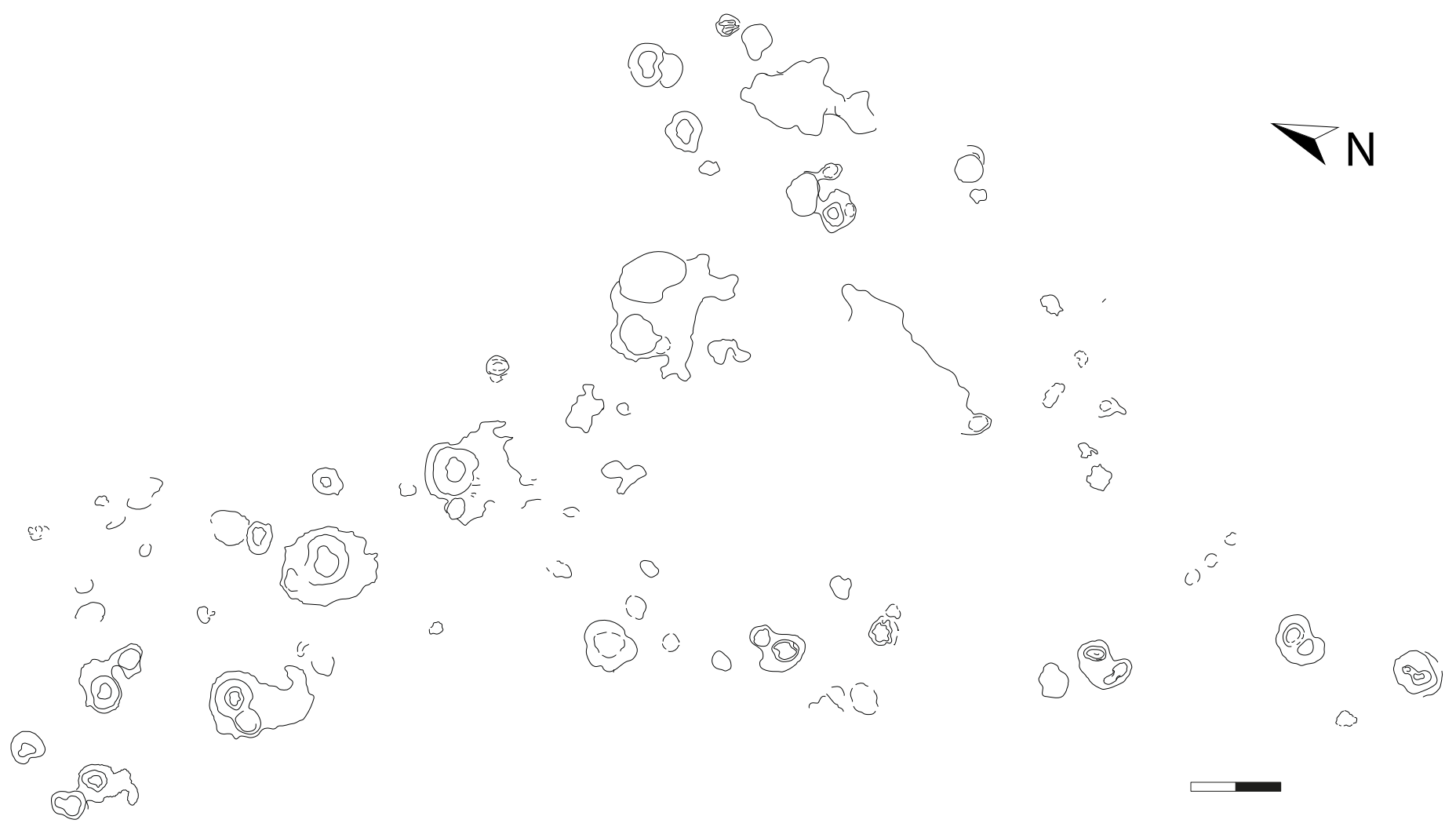

Fig. 4 - Esempio di rilievo icnologico effettuato nell'Area 1. La lunghezza della barra è $1 \mathrm{~m}$. 

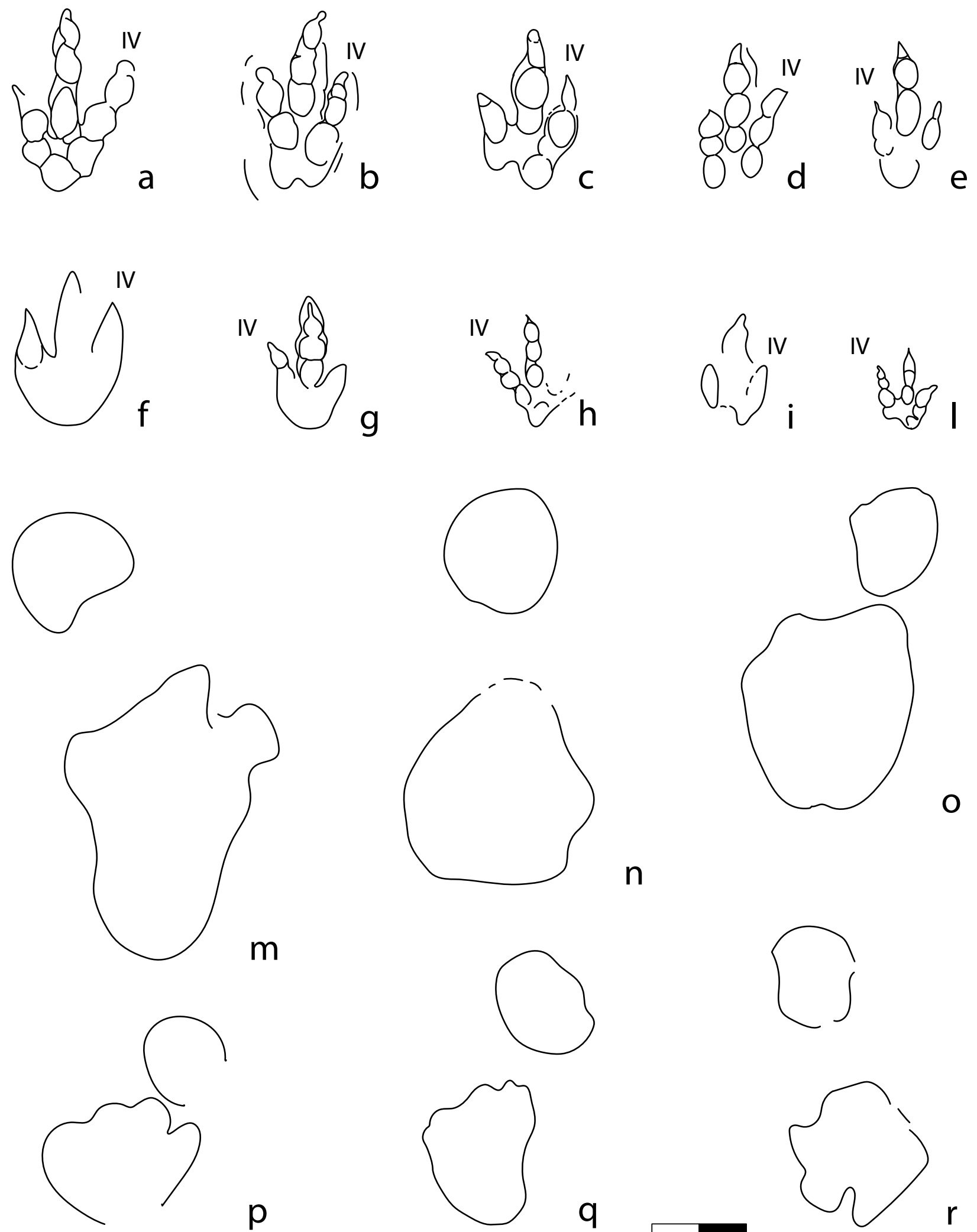

$\mathrm{p}$

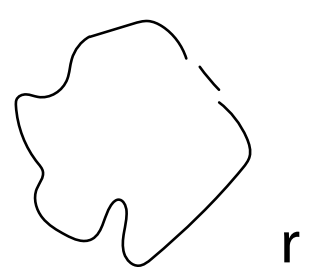

Tavola 3 - a-l) Orme dinosauriane tridattile attribuibili a teropodi di piccole-medie dimensioni. m-o) Coppie manus-pes attribuibili a dinosauri quadrupedi, erbivori e di taglia media (?sauropodi). p-r) Coppie manus-pes di dinosauri quadrupedi, erbivori, riconducibili al gruppo degli anchilosauri. La lunghezza della barra è $20 \mathrm{~cm}$. 

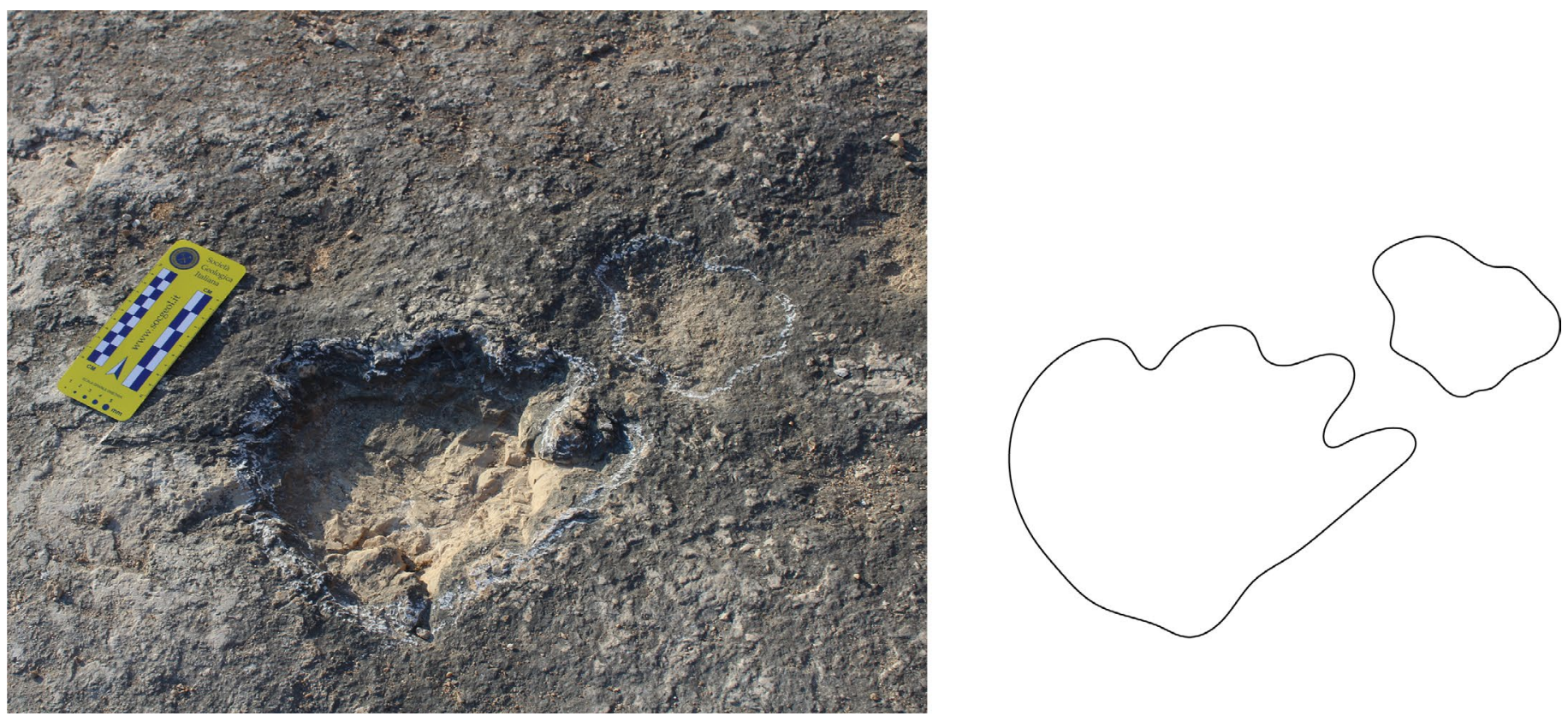

Fig. 5 - Coppia manus-pes di dinosauro quadrupede probabilmente riconducibile al gruppo degli Anchylosauria nell'Area 1.

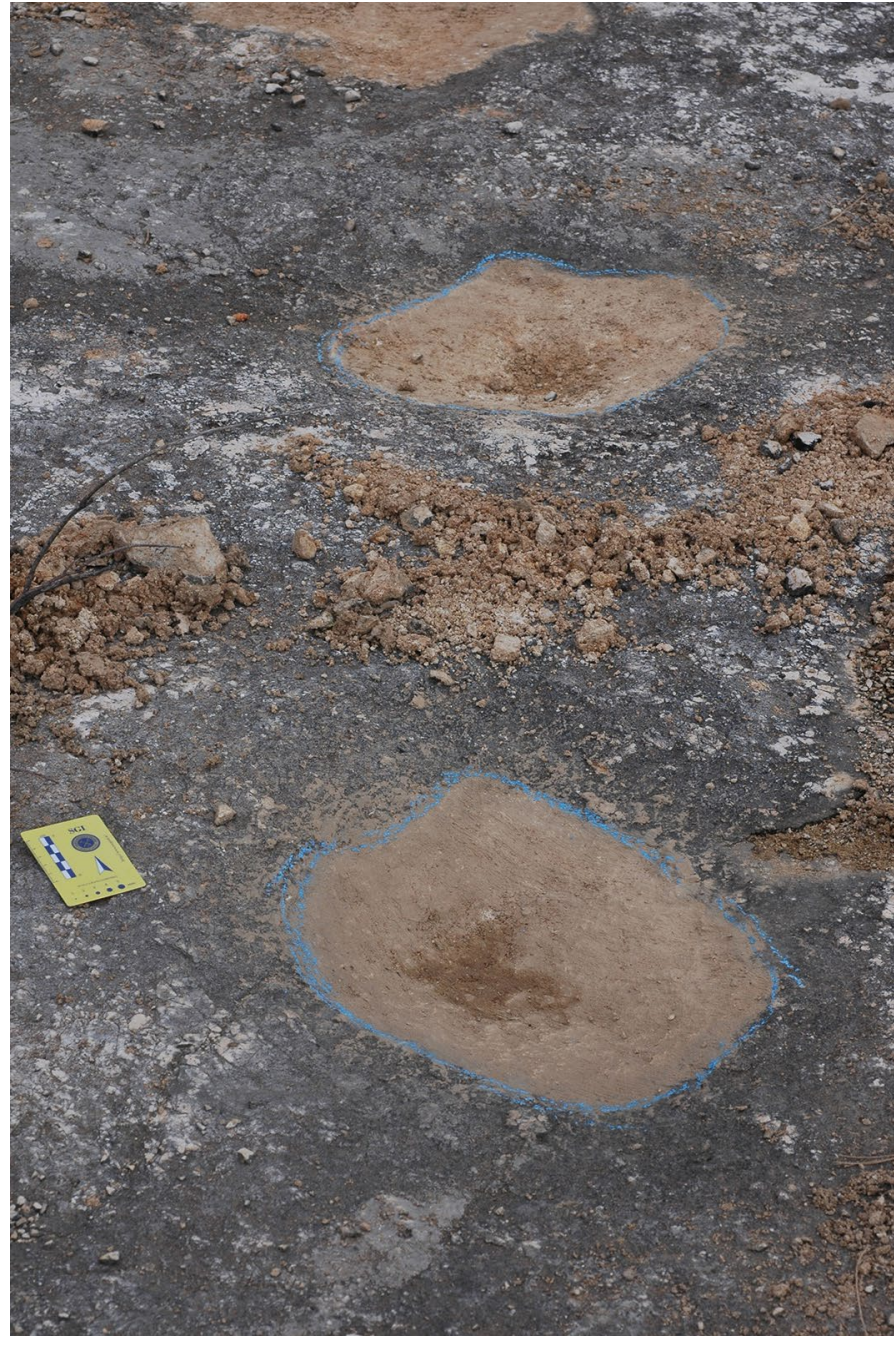

Fig. 6 - Orme di dinosauro di probabile attribuzione al gruppo dei Sauropoda nell'Area 2.
Infine, in entrambe le aree e in diversi altri punti della superficie di fondo cava, sono state osservate alcune orme tridattile isolate, ben conservate ma molto superficiali, profonde pochi millimetri (Fig. 7, Tav. 3a-l). Queste ultime possono essere attribuite al clade dei Saurischia, e con certezza affidabile al gruppo dei Theropoda, dinosauri carnivori ad andatura bipede. La lunghezza delle orme dei teropodi rientra in due classi dimensionali (maggiore e minore di $20 \mathrm{~cm}$ ), indicando una certa variabilità nelle dimensioni di questi animali carnivori.

\section{CONCLUSIONI}

Le orme di dinosauro rinvenute sulla superficie di fondo della ex-Cava SELP ubicata all'interno del "Parco Naturale Regionale Lama Balice" sono di indubbio significato paleontologico, e le osservazioni eseguite su di esse, unitamente alle caratteristiche sedimentarie e di facies ricavate dall'analisi della successione, permettono non solo di attribuire le impronte ad alcuni grandi gruppi di dinosauri, ma anche di fare alcune considerazioni relative ai paleoambienti in cui essi si muovevano.

Per quanto riguarda le orme di dinosauro, la variabilità delle loro caratteristiche in termini di dettaglio morfologico e profondità suggerisce una diversa risposta alla pressione esercitata dagli animali. Molte orme non sono attribuibili ad un preciso trackmaker e non sempre è possibile individuare delle piste anche a causa dell'intenso calpestio (sovrapposizione di orme di più animali). Nonostante ciò, sono state riconosciute alcune orme ben preservate riconducibili ai seguenti trackmaker: i) teropodi; ii) anchilosauri; iii) possibili sauropodi. L'associazione di orme sembra rappresentare un'associazione faunistica a dinosauri relativamente diversificata, comprendendo due taxa di erbivori (anchilosauri, ?sauropodi) e uno di carnivori (teropodi). Tutti i taxa riconosciuti appartengono a dinosauri di piccola-media taglia. 

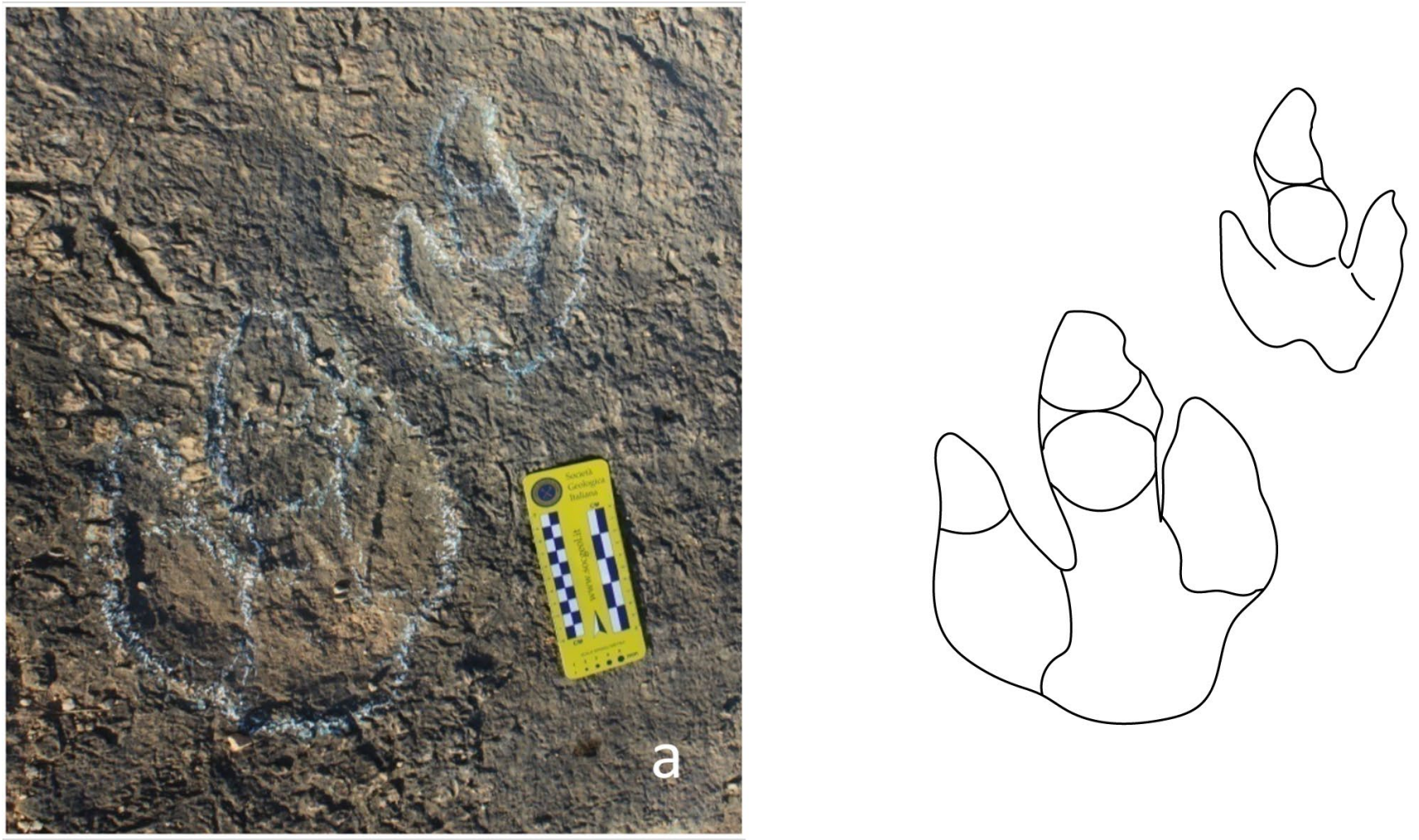

Fig. 7 - Orme tridattile di differenti dimensioni, attribuibili al gruppo dei Theropoda.

Per quanto riguarda l'ambiente deposizionale, sulla base delle osservazioni macroscopiche compiute in cava e di quelle microscopiche sui campioni prelevati, la successione risulta costituita da facies prevalentemente riferibili ad ambienti inter- e supra-tidali. Si può ritenere che la successione studiata si sia sviluppata in ambienti di piana tidale caratterizzata da frequenti esposizioni subaeree.

La presenza di testimonianze geo-paleontologiche di chiaro interesse scientifico e di potenziale richiamo culturale in un'area parco di facile accessibilità e prossima ad una grande città rende le superfici ad orme e le successioni che le ospitano un'eccezionale palestra didattica difficile da ritrovare in altre località del territorio nazionale. Il sito è inoltre adiacente all'aeroporto di BariPalese Karol Wojtyla e può essere raggiunto in 5 minuti con una navetta. Nel solo mese di agosto 2019, l'aeroporto ha registrato 300.000 transiti internazionali. Qualora si riuscisse a intercettare, anche solo in minima parte, questo flusso turistico, il Parco Naturale Regionale Lama Balice potrebbe offrire le sue peculiarità a un importante bacino d'utenza. La presenza di un centro visite (Villa Framarino), già attrezzato ad ospitare turismo culturale e didattico, potrebbe rappresentare la prima tappa di un breve e facile percorso natura. Questo si potrebbe sviluppare lungo la Lama Balice e, dopo alcune centinaia di metri, condurre alla superficie di cava caratterizzata dalle orme di dinosauro. Queste ultime rappresenterebbero il richiamo internazionale per spingere i turisti di passaggio a dedicare parte del loro tempo di transito in un'attività culturale. Un auspicato aumento di questo tipo di visitatori permetterebbe di impegnare anche in momenti stagionali non dedicati al turismo scolastico il personale dedito alla divulgazione.

Tutte queste caratteristiche rappresentano un'ulteriore conferma che il sito di Lama Balice debba essere considerato un geosito di rilevanza nazionale, essendo: i) una rara testimonianza dell'ambiente che nel Cretaceo ha caratterizzato il tratto della piattaforma carbonatica che oggi rappresenta l'ossatura del rilievo murgiano; ii) un potenziale attrattore scientifico, didattico e culturale ubicato in una posizione strategica da un punto di vista logistico.

\section{RINGRAZIAMENTI}

Gli Autori desiderano ringraziare l'Ente Parco Naturale Regionale Lama Balice e la Soprintendenza Archeologia, Belle Arti e Paesaggio per la Città Metropolitana di Bari con i quali l'Università degli Studi di Bari Aldo Moro ha stipulato una Convenzione che ha avuto lo scopo di studiare la superficie ad orme di dinosauro, oggetto di questo lavoro. Un ringraziamento va inoltre alla Fondazione Puglia per il finanziamento concesso al Dipartimento di Scienze della Terra e Geoambientali dell'Università di Bari impiegato per l'acquisto di uno strumento per la realizzazione di sezioni sottili di roccia, utilizzate ai fini dell'analisi di facies della successione studiata. Gli Autori ringraziano i Signori Mazzitelli e Robles per aver gentilmente concesso il permesso all'accesso dei luoghi di studio.

Si ringrazia ISPRA - Dipartimento per il Servizio Geologico d'Italia nelle persone del Dr. Claudio Campobasso e della Dr.ssa Maria Teresa Lettieri per l'autorizzazione concessa all'uso di una porzione del $F^{\circ} 438$ "Bari".

Gli autori ringraziano infine il Prof. Giuseppe Leonardi e il Prof. Ismar de Souza Carvalho per l'utile confronto sul terreno, il Prof. Marco Brandano ed un referee anonimo per la lettura critica del manoscritto e per gli utili suggerimenti che hanno contribuito a migliorarlo, l'editor in chief Prof. Domenico Calcaterra e l'associate editor Prof.ssa Francesca Cifelli per la loro grande pazienza editoriale. 


\section{BIBLIOGRAFIA}

Alexander R. MCN. (1976) - Estimates of speeds of dinosaurs. Nature, 261, 129-130.

Andriani G. \& Walsh N. (2009) - An example of the effects of anthropogenic changes on natural environment in the Apulian karst (southern Italy). Environ. Geol., 58, 313-325.

Azzaroli A. \& Valduga A. (1967) - Note illustrative della Carta Geologica d'Italia. Foglio 177 "Bari" e Foglio 178 "Mola di Bari". Serv. Geol. d'It., 26 pp.

Bosellini A. (2002) - Dinosaurs "re-write" the geodynamics of the eastern Mediterranean and the paleogeography of the Apulia Platform. Earth-Science Review, 59, 211-234.

Cardia S. (2019) - Rilievo digitale di un sito ad orme di dinosauro della Piattaforma Carbonatica Pugliese (Lama Balice, Bari). Tes di Laurea inedita, Università degli Studi di Bari Aldo Moro, 89 pp.

Ciaranfi N., Pieri P. \& Ricchetti G. (1988) - Note alla carta geologica delle Murge e del Salento (Puglia centromeridionale). Mem. Soc. Geol. It., 41, 449-460.

Conti M. A., Morsilli M., Nicosia U., Sacchi E., Savino V., Wagensommer A., Di Maggio L. \& Gianolla, P. (2005) - Jurassic dinosaur footprints from Southern Italy: footprints as indicators of constraints in paleogeographic interpretation. Palaios, 20(6), 534-550.

D'Argenio B. (1974) - Le piattaforme carbonatiche periadriatiche Una rassegna di problemi nel quadro geodinamico mesozoico dell'area mediterranea. Mem. Soc. Geol. It., 13, 137-160.

D’Argenio B., Pescatore T. \& Scandone P. (1973) - Schema geologico dell'Appennino meridionale (Campania e Lucania). In: "Moderne vedute sulla geologia dell'Appennino”. Acc. Naz. dei Lincei, Quad. 183, 49-72.

Dunham R.J. (1962) - Classification of carbonate rocks according to depositional texture, In: HAM W.E. - Classification of carbonate rocks. AAPG Mem., 1, 108-121.

Francescangeli R., La Perna R., Marino M., Marsico A., Petruzzelli M., Petti F., Sabato L., Spalluto L. \& Tropeano M. con la collaborazione di: Cardia S., Carlucci F., Cilumbriello A., Mangione N., Montrone G. \& Stigliano E. (2019) - Lama Balice - Piano di primo intervento per lo studio e la conservazione di alcune superfici di interesse culturale geo-paleontologico. Relazione finale, Convenzione per lo studio e la conservazione di alcune superfici di interesse culturale geo-paleontologico - Università degli Studi di Bari Aldo Moro d'intesa con Soprintendenza per i beni archeologici della Puglia e Parco Naturale Regionale Lama Balice, 77 pp.

Gioia D., Sabato L., Spalluto L. \& Tropeano M. (2011) - Fluvia landform in relation to the geological setting in the "Murge Basse" karst of Apulia (Bari Metropolitan Area, Southern Italy). Journal of Maps, 148-155. https://doi.org/10.4113/jom.2011.113

Iannone A. (2003) - Facies analysis of Upper Cretaceous peritidal limestones characterized by the presence of Dinosaur tracks (Altamura, southern Italy). Mem. Scienze Geologiche, 55, 1-12.

Lockley M.G. \& Conrad K. (1989) - The Paleoenvironmental Context, Preservation and Paleoecological Significance of Dinosaur Tracksites in the Western USA. In: Gillette D.D. \& Lockley M.G. (eds) Dinosaur Tracks and Traces. Cambridge University Press, Cambridge, 121-134.

Lockley M.G., Farlow J.O. \& Meyer C.A. (1994) - Brontopodus and Parabrontopodus ichnogen. nov. and the significance of wide-and narrow-gauge sauropod trackways. Gaia, 10, 135-145.

Mastronuzzi G., Valletta S., Damiani A., Fiore A., Francescangeli R., Giandonato P.B., Iurilli V., Sabato L. (2015) - Geositi della Puglia. Graphic Concept Lab, Bari, 394 pp..

Mongelli G., Buccione R. \& Sinisi R. (2015) - Genesis of autochthonous and allochthonous Apulian karst bauxites (Southern Italy): Climate constraints. Sedimentary Geology, 325, 168-176.

Moretti M. (2005) - Le alluvioni nel settore adriatico delle Murge (terra di Bari): cause geologiche e ruolo dell'azione antropica. Geologi e territorio, 3, 11-22.

Mossa M. (2007) - The floods in Bari: What history should have tought. Jour. of Hydraulic Research, 5, 579-594.

Nicosia U., Marino M., Mariotti N., Murano C., Panigutti S., Petti F. M. \& Sacchi E. (2000a) - The Late Cretaceous Dinosaur tracksite near Altamura (Bari, Southern Italy). I - geological framework. Geologica Romana, 35, 231-236.
Nicosia U., Marino M., Mariotti N., Murano C., Panigutti S., Petti F. M. \& Sacchi E. (2000b) - The Late Cretaceous Dinosaur tracksite near Altamura (Bari, Southern Italy). II - Apulosauripus federicianus new ichnogen. and new ichnosp. Geologica Romana, $35,237-247$.

Nicosia U., Petti F.M., Perugini G., D’Orazi Porchetti S., Sacchi E., Conti M.A., Mariotti N. \& Zarattini A. (2007) - Dinosaur tracks as paleogeographic constraints: New scenarios for the Cretaceous geography of the Periadriatic region. Ichnos, 14, 69-90, https:// doi.org/10.1080/10420940601006859

Petruzzelli M. (2008) - Orme di dinosauro nella Formazione del Calcare di Bari, Aptiano delle Murge baresi Nord-Orientali. Rilievo 3D con Laser Scanner. Tesi di Laurea inedita, Università di Bari, 133 pp.

Petruzzelli M. (2017) - Studio stratigrafico-sedimentologico di alcuni intervalli di interesse regionale o globale nella successione albiano-cenomaniana della Piattaforma Carbonatica Apula. Tesi di Dottorato inedita, Università di Bari, $111 \mathrm{pp}$.

Petruzzelli M., Iannone A. \& La Perna R. (2012) - Fossil heritage in Apulia: Mesozoic vertebrates and dinosaur tracksites. Geologia dell'Ambiente, 3, 104-106.

Petruzzelli M., Cardia S., Francescangeli R., La Perna R., Marsico A., Petti F., Marino M., Spalluto L., Sabato L., \& Tropeano M. (2019) - Apulia dinosaur footprints: state of the art and future perspectives. Paleodays 2019 - XIX Edizione delle Giornate di Paleontologia, Benevento/Pietraroja, 21-25 Maggio 2019. Volume degli Abstracts, 40.

Petti F.M. (2006) - Orme dinosauriane nelle piattaforme carbonatiche mesozoiche italiane: sistematica e paleobiogeografia. $\mathrm{PhD}$ Thesis Università degli Studi di Modena e Reggio Emilia, 219 pp.

Petti F. M., Conti M.A., D’orazi Porchetti S., Morsilli M., Nicosia U. \& Gianolla P. (2008) - A theropod dominated ichnocoenosis from late Hauterivian-early Barremian of Borgo Celano (Gargano Promontory, Apulia, southern Italy). Rivista Italiana di Paleontologia e Stratigrafia, 114(1), 3-17.

Petti F.M., D’orazi Porchetti S., Sacchi E., \& Nicosia U. (2010) - A new purported ankylosaur trackway in the Lower Cretaceous (lower Aptian) shallow-marine carbonate deposits of Puglia, southern Italy. Cretaceous Research, 31(6), 546-552.

Petti F.M., Petruzzelli M., Conti J., Spalluto L., Wagensommer A., Tropeano M., Sabato L., Lamendola M., Francioso R. \& Montrone G. (2018) - The use of aerial and close-range photogrammetry for the study of dinosaur tracksites: the case of the Lower Cretaceous (upper Aptian/lower Albian) Molfetta ichnosite, Bari, Southern Italy. ISSN:1094-8074 Paleontologia Electronica, 31(3), 1-18, https://doi.org/10.26879/845

Pieri P. (1980) - Principali caratteri geologici e morfologici delle Murge. Murgia sotterranea, 2/2, 13-19

Pieri P., Festa V., Moretti M. \& Tropeano M. (1997) - Quaternary tectonic activity of the Murge area (Apulian foreland, southern Italy). Annali di Geofisica, 40,1395-1404.

Pieri P., Sabato L., Spalluto L. \& Tropeano M. (2011a) - Note illustrative della carta geologica dell'area urbana di Bari in scala 1:25.000. Rend. Online Soc. Geol. It., 14, 26-36.

Pieri P., Spalluto L., Sabato L. \& Tropeano M., Con Contributi Di: Andriani G., Caffau M., Labriola M., Maggiore M., Marino M. \& Walsh N. (2011b) - Note illustrative della Carta Geologica d'Italia alla scala 1:50.000, Foglio 438 Bari. ISPRA, Serv. Geol. d'It., Ed. LAC, ISBN: 9788824029902, 105 pp. http://www.isprambiente. gov.it/Media/carg/note_illustrative/438_Bari/.pdf

Ricchetti G. (1975) - Nuovi dati stratigrafici sul Cretaceo delle Murge emersi da indagini nel sottosuolo. Boll. Soc. Geol. It., 94 (3), 1013 1108.

Ricchetti G., Ciaranfi N., Luperto Sinni E., Mongelli F. \& Pieri P. (1988) - Geodinamica ed evoluzione sedimentaria e tettonica dell'avampaese apulo. Mem. Soc. Geol. It., 41, 57-82.

Sacchi E., Conti M.A., D’Orazi Porchetti S., Logoluso A., Nicosia U., Perugini G. \& Petti F. (2009) - Aptian dinosaur footprints from the apulian platform (Bisceglie, Southern Italy) in the framework of periadriatic ichnosites. Palaeogeogr. Palaeoclimatol. Palaeoecol. 271, 104-116.

Servizio Geologico d'Italia (2011) - Carta geologica in scala 1:50.000, Foglio Geologico 438 Bari. ISPRA, Roma. Ed. LAC. ISBN 9788824029902. http://www.isprambiente.gov.it/Media/carg/438 BARI/Foglio.html 
Spalluto L. (2012) - Facies evolution and sequence chronostratigraphy of a mid-Cretaceous shallow-water carbonate succession of the Apulia Carbonate Platform from the Northern Murge area (Apulia, southern Italy). Facies, 58, 17-36.

Spalluto L. \& Caffau M. (2010) - Stratigraphy of the mid-Cretaceous shallow-water limestones of the Apulia Carbonate Platform (Murge, Apulia, southern Italy). Boll. Soc. Geol. It., 129 (3), 335 352.

Spalluto L., Pieri P. \& Ricchetti G. (2005) - Le facies carbonatiche di piattaforma interna del Promontorio del Gargano: implicazion paleoambientali e correlazioni con la coeva successione delle Murge (Italia meridionale, Puglia). Boll. Soc. Geol. It., 124, 675 690.
Stigliano E. (2019) - Caratteri stratigrafici e sedimentologici della successione albiana del Calcare di Bari contenente una superficie a orme di dinosauro nella ex-cava SELP (Parco Lama Balice, Città Metropolitana di Bari). Tesi di Laurea inedita, Università degli Studi di Bari Aldo Moro, 78 pp.

Valduga A. (1965) - Contributo alla conoscenza geologica delle Murge baresi. Studi geologici e geomorfologici sulla regione pugliese, 1, $1-14$.

Zarcone G., Petti F.M., Cillari A., Di Stefano P., Guzzetta D. \& Nicosia U. (2010) - A possible bridge between Adria and Africa: New palaeobiogeographic and stratigraphic constraints on the Mesozoic palaeogeography of the Central Mediterranean area. Earth-Science Reviews, 103, 154-162. 